\title{
A MEMS-based tactile sensor to study human digital touch: mechanical transduction of the tactile information and role of fingerprints
}

\author{
J. Scheibert ${ }^{12, a}$, G. Debrégeas ${ }^{1}$, and A. Prevost ${ }^{1}$ \\ 1 Laboratoire de Physique Statistique, CNRS/ENS/University Paris 6/University Paris 7, Paris, France \\ 2 Physics of Geological Processes, University of Oslo, Oslo, Norway
}

\begin{abstract}
We present recent results showing that human epidermal ridges (fingerprints) could play a central role in fine texture discrimination tasks by spatially modulating the contact stress field between the fingertip and the substrate. Using an original biomimetic finger whose surface is patterned with parallel ridges, we demonstrate that the subsurface stress signals elicited by continuous rubbing of randomly textured substrates is dominated by fluctuations at a frequency defined by the inter-ridge distance divided by the rubbing velocity. In natural exploratory conditions, this frequency matches the best frequency of one type of mechanoreceptors, namely the Pacinian corpuscles, which are specifically involved in the tactile coding of fine textures. The use of white-noise patterned stimuli has alloowed us to extract, using a reverse-correlation analysis, the stimulus-signal response function associated with roughness modality. Its shape could provides spectral, spatial and directional selectivity to the digital tactile system. It offers a physiological basis for the recently proposed hypothesis of a dual-coding (spatio-temporal and vibratory) of tactile information.
\end{abstract}

\section{Introduction}

The hand is an essential tool for human interaction with its surroundings [1-3]. The high tactile sensitivity of the fingertips allows for many complex tasks, including precise grasping and manipulation of objects, detection of individual defects on smooth surfaces and texture discrimination. The digital skin exhibits characteristic epidermal ridges (fingerprints) that have long been suspected to improve tactile perception, although the mechanism at play remains poorly understood [4]. They are believed to enhance friction and adhesion of the finger pads thus improving the ability to securely grasp objects or supports [5]. It is now clear that fingerprints modifiy significantly the subcutaneous stress [6-9]. In this respect, individual ridges may enhance tactile sensitivity by magnifying subsurface strains $[10,11]$. The latter are further converted into neural signals by a population of mechanosensitive fibers innervating the distal finger pads [12-15], giving rise to tactile sensation at the cortex level. It has long been recognized that tactile performance is improved when the finger is allowed to move relative to the substrate. Very recently, it has been shown that two independent neural coding mechanisms are involved in this dynamic exploratory mode, which might be associated with separate coding channels [16-18]: for textural features of lateral extension greater than about 200 micrometers, a spatio-temporal coding involving slowly adapting mechanoreceptors (sensitive to a constant stimulus) is used [19] whereas finer features are vibratory encoded principally through (rapidly adapting) Pacinian fibers [18,20-23].

In order to understand the performance of tactile perception and the associated pre-neural coding strategy, one needs to describe the process by which the friction of a textured substrate induces the deformation of the dermis eliciting the neural signals. In particular, one may ask which features

a e-mail: julien.scheibert@fys.uio.no 
of the substrate properties survive this pre-neural transduction process. Since the skin is intrinsically diffusive to light, experiments performed on real fingers have been restricted to global force measurements. Local subsurface measurements are only accessible via biomimetic approaches where the finger is replaced by an artificial sensor whose characteristics match those of the physiological system. Another difficulty owes to the design of stimuli substrates: so far, they have consisted either of mechanical actuators of millimetric dimensions, or of regularly spaced dots or bars, of different characteristic sizes, moved across the finger pad. Although they provide information on the receptive fields of the mechanoreceptors, i.e. the skin area within which they respond, they are spectrally too poor to allow for a complete description of the vibratory transduction process. In a recent study [24], we directly addressed the question of the tactile transduction and the role played by fingerprints using a biomimetic finger equipped with either a smooth or a fingerprinted skin. Combined with engineered random stimuli substrates, it allowed us to implement a reverse correlation analysis to the pre-neural stage of tactile transduction.

\section{Methods}

\subsection{Skin patterning and substrates engineering}

Smooth and fingerprinted skins of maximal thickness $2 \mathrm{~mm}$ were made out of an optically transparent PDMS elastomer (PolyDimethylSiloxane, Sylgard 184) of elastic modulus 2.2 $\pm 0.1 \mathrm{MPa}$ (Fig. 1). Their spherical shape was obtained by filling, prior to curing, a plano-concave spherical glass lens (radius of curvature $128.8 \mathrm{~mm}$ ) with the liquid PDMS-crosslinker mixture and then glued on top of the micro-sensor using a thin PDMS-crosslinker liquid film. To design the fingerprinted skin, soft photolithography was used. A layer of photoresist (SU8-2035) was spin-coated on the lens, and UVexposed through a mask consisting of alternating opaque and transparent parallel stripes of width 110

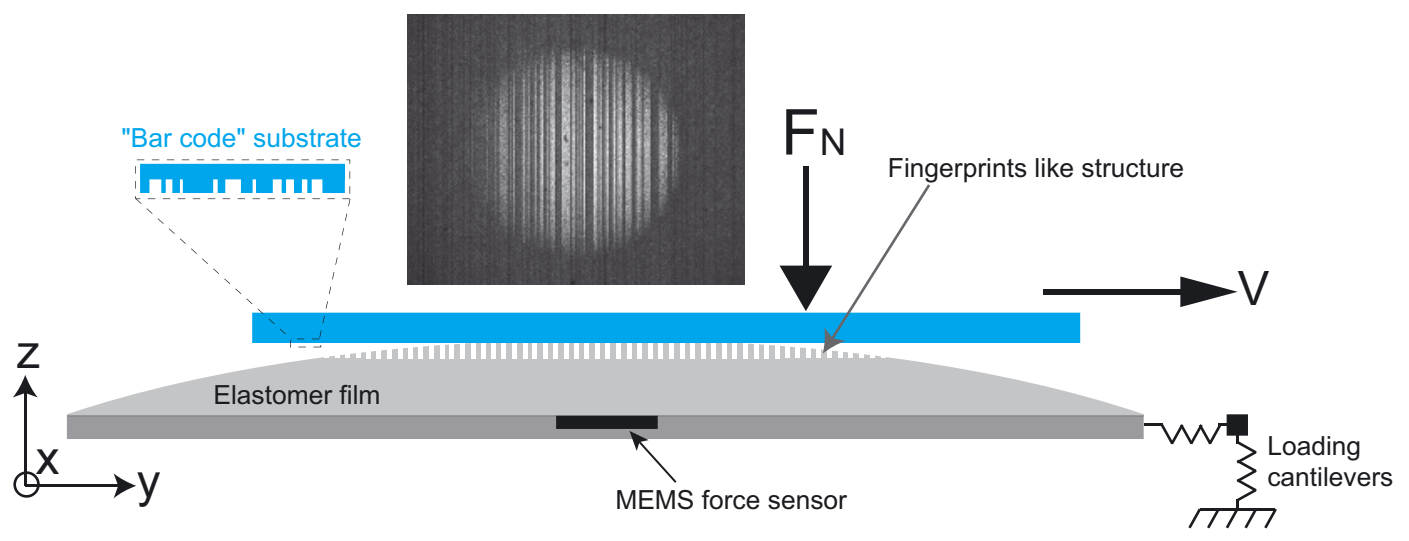

Fig. 1. Sketch of the experimental setup. The biomimetic finger is made of a MEMS micro-force sensor solidly attached to a rigid base (dark grey) and embedded in a spherical elastomer cap (light grey). The cap's surface is either smooth or patterned with equally spaced parallel ridges as a model for fingerprints. The biomimetic finger is mounted on a system of two cantilevers to prescribe and measure both normal and tangential loads. When pressed under a constant normal load $F_{N}$ against a nominally flat glass substrate covered with a photoresist pattern (blue), one obtains a circular contact region (typical area $1 \mathrm{~cm}^{2}$ ) divided into parallel stripes of real contact (bright regions on the top image). This picture was obtained by imaging in transmission an analogous transparent contact [25]. Local and global forces fluctuations are recorded upon sliding. 
$\mu \mathrm{m}$. After development, one was left with a grating pattern of parallel grooves $28 \mu \mathrm{m}$ deep, thus producing an elastomer cap with regularly spaced ridges (spatial period $220 \mu \mathrm{m}$ ). Both skins display a mat finish (a random micrometric roughness) in order to reduce their adhesion energy.

Textured heterogeneous substrates were made using the same lithography techniques. Structures infinitely rigid as compared to the elastomer skin were produced by patterning $28 \mu \mathrm{m}$ thick layers of SU8-2035 photoresist spin coated on glass slides. The masks were designed with a bar code like pattern (see Fig. 1) consisting of successive and alternating parallel opaque and transparent stripes. Their width was determined by randomly choosing from a uniform distribution the positions of their edges. This procedure, applied for various mean distances $\lambda_{e}$ between edges, produced topographies whose power spectrum was that of a white noise with a cut-off spatial frequency $1 / \pi \lambda_{e}$. To further reduce adhesion to a non-measurable quantity with our setup, both skins and rough substrates were eventually silanized by exposing them for 12 hours to a $1 \mathrm{H}, 1 \mathrm{H}, 2 \mathrm{H}, 2 \mathrm{H}$-Perfluorodecyltrichlorosilane vapour phase.

\subsection{Experiments}

Experiments were carried out using a frictional setup described elsewhere [26-28] and sketched on Fig. 1, which allows putting into contact under a constant prescribed normal load $F_{N}$ both biomimetic fingers against the substrates, and pulling the formers at a constant velocity $V$ using a DC linear motor. The biomimetic fingers consist of a micro force sensor glued to a rigid metallic base and covered with the adequate elastomer skin. They are mounted on a double cantilever system allowing to measure simultaneously the normal and tangential loads using capacitive position sensors. The micro force sensor is a MEMS sensor, with a sensitive part of size $500 \mu \mathrm{m}$, designed by LETI (CEA, Grenoble, France). It produces voltage outputs proportional to the local normal force and shear forces along two transverse directions. Calibration to relate its voltages to stress measurements once it is embedded in the elastomer was done previously [26,27], using point indentation of the skin surface. Precision translation stages allow for micrometric positioning of the biomimetic fingers with respect to the substrates.

\section{Results}

Figure 2 shows the comparison of pressure signals $p$ obtained with both types of skin rubbed against different substrates. The applied load is $F_{N}=1.71 \mathrm{~N}$ and the sliding velocity is $V=0.2 \mathrm{~mm} / \mathrm{s}$. For such moderate velocities, the signals are found to be independent of $V$ and are thus plotted as a function of the sample position $u$. To facilitate comparison between both fingers, $p$ is normalized by its time-average $\langle p\rangle$. As a reference, the first panel (Fig. 2a) shows the results obtained with a smooth silanized substrate for which both sensors produce nearly constant signals. The second panel (Fig. 2b) corresponds to random rough substrates. The smooth finger exhibits pressure fluctuations with a characteristic wavelength in the millimeter range. The fingerprinted sensor produces similar long-range modulations on which are superimposed large oscillations of characteristic wave-length equal to the inter-ridge distance $\lambda=220 \mathrm{~m}$. The power spectra of the signals elicited by the rough substrate are displayed in Fig. 2c for the smooth skin and Fig. 2d for the fingerprinted skin. Both fingers act as a low-pass filter. However, the fingerprinted sensor also exhibits band-pass filtering around the spatial frequency $1 / \lambda$ with further harmonics amplifications at integer multiples of $1 / \lambda$. The relative maximum amplification induced by the ridges is of the order of 100 .

To further characterize the filtering properties of both skins, i.e. to relate the measured pressure signal $p$ to the topography stimulus $T$, we used a reverse-correlation technique. If $p(u)=G[T(x)]$, one can always write the following Wiener/Volterra representation [29-31]:

$$
p(u)=p_{0}+\int G_{1}(x) T(x-u) d x+\iint G_{2}\left(x_{1}, x_{2}\right) T\left(x_{1}-u\right) T\left(x_{1}-u\right) d x_{1} d x_{2}+\ldots
$$

When $T$ is a normalized Gaussian white noise, the first (linear) function $G_{1}$ can be evaluated by cross-correlating the signal $p$ and the stimulus $T$. The linearized response function is thus experimentally obtained for both smooth and fingerprinted skin as displayed in Fig. 3. The error bars indicate 

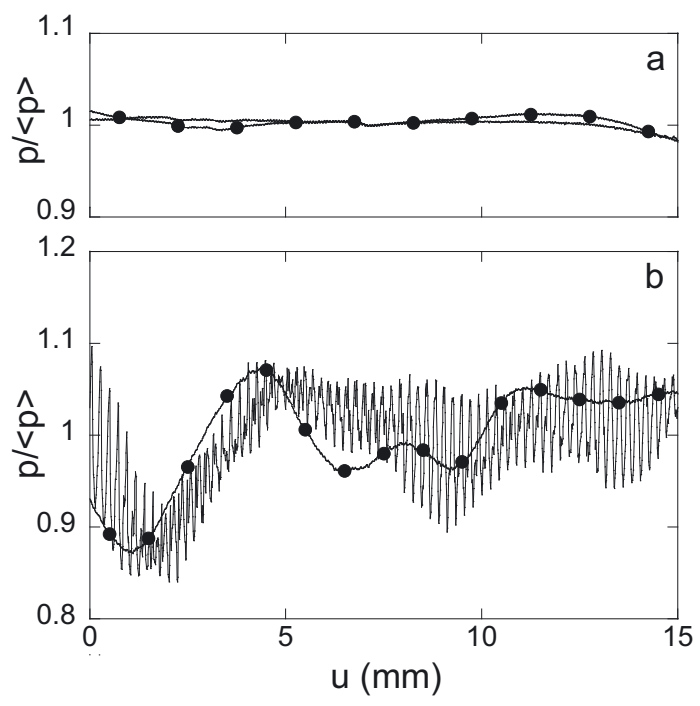
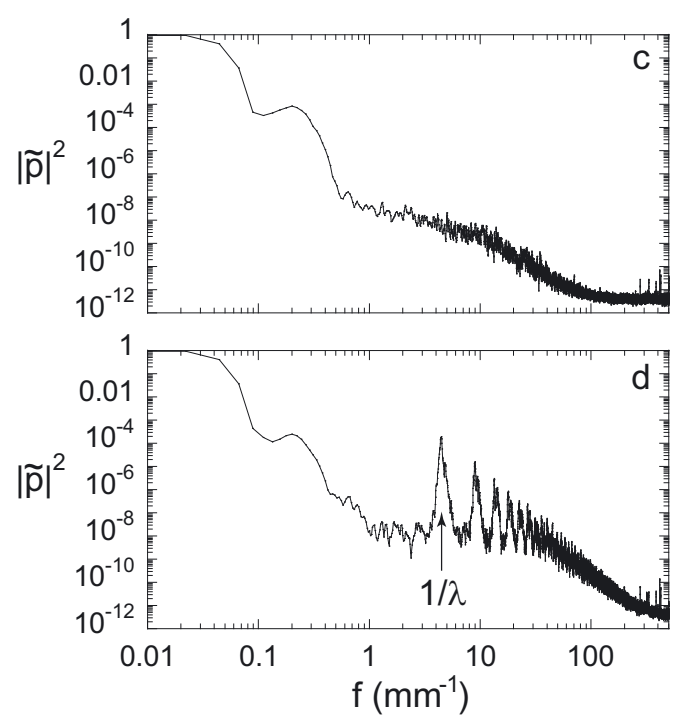

Fig. 2. Left: Local pressure fluctuations $p$ normalized by their time average $\langle p\rangle$ as a function of the spatial displacement $u$, at $P=1.71 \mathrm{~N}$ and for different types of substrates. Solid lines with the superimposed solid disks show the signals obtained with the smooth biomimetic finger, whereas those measured with the fingerprinted sensor are represented by the solid lines. (a) Smooth silanized photoresist substrate. (b) Rough heterogeneous substrate with $\lambda_{e}=75 \mu \mathrm{m}$. Right: Power spectra of the two pressure signals represented in (b). (c): smooth skin. (d) fingerprinted skin.

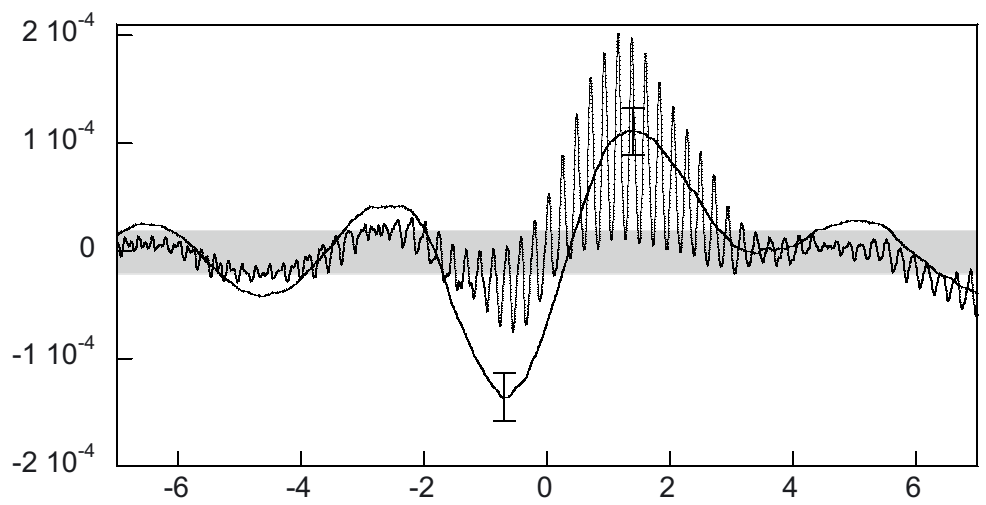

Fig. 3. Linearized stimulus-signal response functions $G 1(x)$ computed by cross-correlating the pressure signals and the stimulus topography $T(x)$, for both smooth and fingerprinted skins. The expected statistical deviation due to the finite length of the substrates is shown with the error bars and the shaded rectangle.

the statistical deviations numerically estimated using the experimental parameters. The smooth skin response function is odd symmetrical with a width of order $2 \mathrm{~mm}$. A comparable overall shape is observed with the fingerprinted skin, but the response function is modulated with a spatial frequency $1 / \lambda$. It should be stressed that changing the overall pressure does not only modify the scaling factor, but also significantly transforms the shape of the response functions. This response function field can be viewed as a tactile Gabor filter since it provides both spatial and spectral resolution, set by the sensor depth $h$ and $1 / \lambda$ respectively. Such filters are classically used in image analysis, and have been identified in visual system at the neural level [32]. They are known to provide orientation discrimination, contrast enhancement, motion detection and texture discrimination. 
Such response functions are qualitatively reproduced by a simple linear model of textured contact mechanics. Following Howe and Cutkosky's general analysis of biomimetic fingers [33], we consider a small linear force sensor embedded at a depth $h$ in an elastic skin. By assuming that the interfacial stress field $\sigma_{s}(x)$ against a smooth substrate simply becomes $2 T(x) \sigma_{s}(x)$ for the binary rough substrates considered here, one can show that the following reltationship holds [24]:

$$
G_{1}(x)=\int\left(F \cdot \sigma_{s}\right)(x, y) d y,
$$

where $F$ is the receptive field of the sensor.

The two terms in the integral correspond to two different aspects of the system whose combination controls the transduction properties. The receptive field $F$ is related to the intrinsic bulk property of the organ. It can be measured by applying unit stress impulses at different locations on the free surface of the skin and has a lateral width of order $h$. One should notice that if the force sensor dimension and the skin roughness (the depth of epidermal ridges for instance) are smaller than the depth $h$, then $F$ is expected to be independent of the skin texture [34]. In contrast, $\sigma_{s}$ depends on the applied normal load, the friction coefficient and the location of the sensor with respect to the contact, which are extrinsic parameters. Moreover, short-scale variations of $\sigma_{s}$ are controlled by the surface texture such as epidermal ridges.

The shape of the observed response functions of Fig. 3 was found in reasonable agreement with the results of a semi-analytical calculation of $G_{1}$, taking explicitely into account the exploratory conditions (friction coefficient and normal force) and the location of the MEMS sensor within the contact.

\section{Discussion and conclusions}

At this point, a legitimate question is whether the vibrations evidenced with this idealized device are relevant to an actual fingertip. Answering this question poses a technical challenge since there is no current way to measure the stress experienced by mechanoreceptors in an actual finger. However, one may expect that differences in texture-induced subcutaneous vibrations may show up in the global friction force acting on the finger during tactile exploration. This idea is at the basis of recent experiments in which the vibrations of the fingertip skin were locally measured with a displacement probe as the finger was scanned across various textured substrates [18]. The intensity of these vibrations weighted by the Pacinian's spectral sensitivity could be correlated with the perception of roughness determined independently through psychophysical experiments [35].

Along a similar line, we have designed a set-up which allows one to monitor the tangential force $F_{T}$ acting on a flat substrate as it is rubbed against a fingertip at constant speed and normal load [36]. The stimuli substrates were the same as the ones used with the biomimetic sensor. The forefinger was constrained in a fixed position at $45^{\circ}$ with respect to the substrate plane. By comparing the force signal obtained upon moving the substrate distally (mode 1, orthogonal to fingerprints) versus radially (mode 2 , parallel with fingerprints), we could directly probe the impact of fingerprints in shaping the skin vibrations spectrum. In mode 1 , the vibrations signals exhibited characteristic oscillations at a spatial frequency close to $1 / \lambda_{r}$ where $\lambda_{r} \simeq 500 \mu \mathrm{m}$ is the interridge distance for a real human fingertip. Over the range of loads explored, the relative amplification induced by the fingerprints was of the order of 100 , like on the biomimetic system. In mode 2 , no oscillation was observed.

The tactile sensor used in this study offers a crude version of the finger physiology [37-39]. However, certain aspects of our results should be sufficiently robust against the details of the system so that it is worth discussing their consequence on human digital touch. First, we showed that the presence of epidermal ridges, by chopping the interfacial stress field, strongly modifies the pre-neural filtering properties of the finger. As compared to a smooth skin, the fingerprints selectively amplifies the signal around a frequency $f_{0}=V / \lambda$, where $V$ is the relative finger/substrate velocity and $\lambda$ the inter-ridge distance. In natural exploratory conditions, $V$ is of order $10-15 \mathrm{~cm} / \mathrm{s}$ and $\lambda=\lambda_{r} \simeq 500 \mu \mathrm{m}$ so that $f_{0}=200-300 \mathrm{~Hz}$. This range corresponds to the best frequency of Pacinian corpuscles (i.e. the stimulus frequency where maximum sensitivity occurs) [40] which are known to be involved in the coding of fine textures. 
In this respect, one might see the development of epidermal ridges as a strategy to shape the signal specifically to the Pacinians. This spatial modulation of the tactile stimulus plays the role, in glabrous touch, of the band-pass filtering and amplification resulting, in other mechano-sensory systems, from the resonant mechanical oscillation of the tactile organ. For instance, in rodents vibrissal touch, the whisker resonance frequency matches the response of the mecano-receptors located in the muzzles [41]. In auditory perception, the cochlea resonance locally matches the frequency of hair cells [42].

Our results might be a source of inspiration in the field of robotics, in particular with the scope of providing robots with tactile capabilities approaching the ones of humans [43-45]. Most of the current systems used to detect or recognize the topography of substrates rely on tactile devices made of a matrix of force sensors, the period of which (at best of the order of a few hundreds of micrometers) sets the limiting spatial resolution (see e.g. [46-52] for recent examples). We suggest that this limit might be overcome by adding small scale patterns at the surface of the elastic membrane covering the sensors.

\section{References}

1. L.A. Jones, S.J. Lederman, Human Hand Function (Oxford University Press, New York, 2006)

2. R.S. Johansson, J.R. Flanagan, Nature Reviews Neuroscience 10(5), (2009) 345

3. M. Hollins, Annual Review of Psychology 61(1), 243 (2010), ISSN 0066-4308

4. D.Z. Loesch, N.G. Martin, Annals of Human Biology 11(2), (1984) 113

5. M. Cartmill, American Journal Of Physical Anthropology 50(4), (1979) 497

6. T. Maeno, K. Kobayashi, N. Yamazaki, JSME International Journal. Series C, Mechanical systems, machine elements and manufacturing 41(1), (1998) 94

7. D. Yamada, T. Maeno, Y. Yamada, Journal on Robotics and Mechatronics 14, (2002) 140

8. G.J. Gerling, G.W. Thomas, The Effect of Fingertip Microstructures on Tactile Edge Perception, in First Joint Eurohaptics Conference and Symposium on Haptic Interfaces for Virtual Environment and Teleoperator Systems (WHC'05), pp63-72 (2005)

9. G. Vásárhelyia, B. Fodorb, T. Roska, Sensors and Actuators A: Physical 140, (2007) 8

10. N. Cauna, Anatomical Record 119(4), (1954) 449

11. R.S. Fearing, J.M. Hollerbach, International Journal Of Robotics Research 4(3), (1985) 40

12. I. Darian-Smith, Handbook of Physiology, Section 1: The nervous system, Volume III (1984), chap. 17: The sense of touch: performance and peripheral neural processes, p. 739

13. K.O. Johnson, J.R. Phillips, Journal of Neurophysiology 46(6), (1981) 1177

14. J.R. Phillips, K.O. Johnson, Journal of Neurophysiology 46(6), (1981) 1192

15. J.R. Phillips, K.O. Johnson, Journal of Neurophysiology 46(6), (1981) 1204

16. D. Katz, The World of Touch (I. E. Krueger, Trans. \& Ed., Hillsdale, NJ: Erlbaum, 1989, original work published 1925)

17. M. Hollins, S.J. Bensmaïa, S. Washburn, Somatosensory and Motor Research 18(4), (2001) 253

18. M. Hollins, S.J. Bensmaia, Canadian Journal of Experimental Psychology 61(3), (2007) 184

19. S.J. Lederman, M.M. Taylor, Perception and Psychophysics 12(5), (1972) 401

20. M.A. Srinivasan, J.M. Whitehouse, R.H. LaMotte, J Neurophysiol 63(6), (1990) 1323

21. C.J. Cascio, K. Sathian, J. Neurosci. 21(14), (2001) 5289

22. S.J. Bensmaia, M. Hollins, Somatosensory And Motor Research 20(1), (2003) 33

23. S.J. Bensmaia, J.C. Craig, K.O. Johnson, J. Neurophysiol. 95(3), (2006) 1783

24. J. Scheibert, S. Leurent, A. Prevost, G. Debrégeas, Science 323, (2009) 1503

25. J. Scheibert, G. Debrégeas, A. Prevost, Arxiv:0809.3188v1 (2008)

26. J. Scheibert, A. Prevost, J. Frelat, P. Rey, G. Debrégeas, EPL 83(3), (2008) 34003

27. J. Scheibert, A. Prevost, G. Debrégeas, E. Katzav, M. Adda-Bedia, Journal of the Mechanics and Physics of Solids 57(12), (2009) 1921

28. J. Scheibert, E. Katzav, M. Adda-Bedia, J. Frelat, A. Prevost, G. Debrégeas, MEMS-based contact stress field measurements at a rough elastomeric layer: local test of Amontons' friction law in static and steady sliding regimes, in International Conference of Experimental Mechanics 14, Poitiers, France, article 474 (2010) 
29. N. Wiener, Nonlinear Problem in Random Theory (MIT Press, 1958)

30. J.J. Eggermont, Hearing Research 66(2), (1993) 177

31. F. Rieke, D. Warland, R. de Ruyter van Steveninck, W. Bialek, Spikes: Exploring The Neural Code (MIT Press, Cambridge, MA, 1999)

32. J.P. Jones, L.A. Palmer, Journal of Neurophysiology 58(6), (1987) 1233

33. R. Howe, M. Cutkosky, IEEE Transactions on Robotics and Automation 9, (1993) 140

34. K.L. Johnson, Contact Mechanics (Cambridge University Press, 1985)

35. S.J. Bensmaia, M. Hollins, Perception and Psychophysics 67(5), (2005) 842

36. A. Prevost, J. Scheibert, G. Debrégeas, Communicative and Integrative Biology 2, (2009) 422

37. K. Dandekar, B.I. Raju, M.A. Srinivasan, Journal of Biomechanical Engineering-Transactions of the ASME 125(5), (2003) 682

38. J.Z. Wu, R.G. Dong, S. Rakheja, A.W. Schopper, W.P. Smutz, Medical Engineering \& Physics 26(2), (2004) 165

39. Q. Wang, V. Hayward, Journal of Biomechanics 40(4), (2007) 851

40. J.C. Makous, R.M. Friedman, C.J. Vierck, Journal of Neuroscience 15(4), (1995) 2808

41. M.A. Neimark, M.L. Andermann, J.J. Hopfield, C.I. Moore, J. Neurosci. 23(16), (2003) 6499

42. C.D. Geisler, From Sound to Synapse: Physiology of the Mammalian Ear (Oxford University Press, 1998)

43. R. Crowder, Science 312(5779), (2006) 1478

44. V. Maheshwari, R.F. Saraf, Science 312(5779), (2006) 1501

45. R.S. Dahiya, G. Metta, M. Valle, G. Sandini, IEEE Transactions on Robotics, (2010) in press

46. F. Castelli, IEEE Transactions on Industry Applications 38(1), (2002) 85

47. K.H. Yu, T.G. Kwon, M.J. Yun, S.C. Lee, KSME International Journal 16(10), (2002) 1222

48. Z.Y. Wen, Y. Wu, Z.Y. Zhang, S.L. Xu, S.L. Huang, Y.L. Li, Sensors and Actuators A-Physical 103(3), (2003) 301

49. A. Kis, F. Kovacs, P. Szolgay, International Journal of Circuit Theory and Applications 34(4), (2006) 517

50. M. Adam, T. Mohacsy, P. Jonas, C. Ducso, E. Vazsonyi, I. Barsony, Sensors and Actuators A: Physical 142(1), (2008) 192

51. N. Wettels, V.J. Santos, R.S. Johansson, G.E. Loeb, Advanced Robotics 22(8), (2008) 829

52. C.M. Oddo, L. Beccai, M. Felder, F. Giovacchini, M.C. Carrozza, Sensors 9(5), (2009) 3161 